\title{
Assessment of paediatric dental guidelines and caries management alternatives in the post COVID-19 period. A critical review and clinical recommendations
}

\author{
M. Al-Halabi ${ }^{1}$ (D) A. Salami ${ }^{1}$ - E. Alnuaimi ${ }^{1}$ - M. Kowash ${ }^{1} \cdot$ I. Hussein ${ }^{1}$
}

Received: 22 April 2020 / Accepted: 7 June 2020 / Published online: 16 June 2020

(c) European Academy of Paediatric Dentistry 2020

\begin{abstract}
Purpose The first aim of this paper is to provide dental professionals caring for children and adolescents during and after the COVID-19 pandemic with a reference to international dental guidelines. The second aim is to suggest minimally invasive treatment alternatives for caries management, minimising the risk of viral cross-infection and offering a safer clinical environment.

Methods An evidence-based pertinent literature search of different electronic databases was performed in addition to leading global dental authorities, royal colleges, and programmes.

Results All guidelines released in response to COVID-19 centred around minimising Aerosol Generating Procedures (AGP) impacting the provision of regular dental treatment of paediatric patients. There was an emphasis on triaging and only treating emergency and urgent cases. Special attention was given to medically compromised children in the guidelines. Detailed guidelines for the dental environment and equipment were given. This paper also summarised the relevant evidence-based guidelines for the use of non-invasive and minimally invasive caries management techniques.

Conclusion Specific recommendations for dental management of paediatric patients during and in the post-COVID-19 era are suggested. Minimisation of AGP procedures, and case-based selection of biological, non-invasive or minimally invasive methods are recommended.
\end{abstract}

Keywords COVID-19 · Paediatric dentistry · Aerosol generating procedures · Biological caries treatment - Atraumatic restorative treatment $\cdot$ Non-restorative caries control

\section{Introduction}

In December 2019, "COVID-19” originated in China as a result of a novel Corona RNA virus (Dong et al. 2020). It has exposed 7 billion humans on our planet to one of the worst and most rapidly expanding pandemics known to the global population since the Spanish Flu pandemic in 1918-19 (Snyder and Ravi 2018). The global spread of COVID-19 has led to mass gathering controls (Ebrahim and Memish 2020; McCloskey et al. 2020), severely slowed down countries' economies, as it infected more than 6.3 million patients

M. Al-Halabi

manal.halabi@mbru.ac.ae

1 Department of Pediatric Dentistry, Hamdan Bin Mohammed College of Dental Medicine (HBMCDM), Mohammed Bin Rashid University of Medicine and Health Sciences (MBRU), P.O. Box 35701, Dubai, UAE including the global paediatric population (Rabaan et al. 2020; Yang et al. 2020; Choi et al. 2020; Cao et al. 2020; Cruz and Zeichner 2020; Dong et al. 2020).

COVID-19 is a Severe Acute Respiratory Syndrome (SARS) caused by SARS-CoV-2 virus (Rabaan et al. 2020; Yu et al. 2020). It is believed to be transmitted via droplet infection or contact with contaminated surfaces (Rothan and Byrareddy 2020). After a period of incubation of up to 14 days, patients may experience a range of no symptoms, or mild symptoms of pyrexia (85\%), dry cough (68.6\%) and general malaise, myalgia or fatigue (35.8\%), expectoration (28.2\%), headache or dizziness (12.1\%), diarrhoea (4.8\%), nausea and vomiting (3.9\%), dyspnea (21.9\%) anosmia and general malaise. Severe symptoms includerespiratory distress and ultimate death (Jiang et al. 2020; Wu et al. 2020). The latter mainly affects those with co-morbidities of all age groups. Detection is achieved by assessment of 
seroprevalence of SARS-CoV-2 antibodies from a nasopharyngeal swab (Rubino et al. 2020).

Paediatric COVID-19 infection is reported to be relatively mild in symptoms when compared to adults and adolescents, and children are also reported to have a better prognosis ( $\mathrm{Su}$ et al. 2020; Shen et al. 2020). Mortality in children appears rare, but those with co-morbidities remain a concern (Dong et al. 2020; Sinha et al. 2020).

Clinical features of COVID-19 in children include fever and cough, but a large proportion of infected children appears to be asymptomatic and may contribute significantly to transmission (Qiu et al. 2020; Dong et al. 2020; Frauenfelder et al. 2020). A specific concern for children is the uncertainty of their infection status; a clinical history may not be as suggestive of the infection as it is in adults (Xia et al. 2020). According to Cruz and Zeichner (2020), 4\% of virologically confirmed cases in children had an asymptomatic infection (Cruz and Zeichner 2020).

SARS-CoV-2 infection seems to have a more benign evolution in children and the infection runs in three major stages: a mild cold-like illness, a moderate respiratory syndrome and a severe acute interstitial pneumonia (Pavone et al. 2020). They found that the median period of viral shedding of COVID-19 was 15 days as measured from illness onset to discharge. This period was shorter in asymptomatic patients compared with symptomatic patients (11 days vs. 17 days). Thus the prolonged duration of viral shedding in children with COVID-19 was associated with symptomatic infection, fever, pneumonia and high lymphocyte count (Zimmerman et al. 2020).

Therefore, it can be assumed that, at this stage in the pandemic, all children and their parents/carers are potentially infective (Royal College of Surgeons England 2020) with the potential of cross-infection to healthcare workers, parents and the public. This has, in theory, an impact on medical and dental procedures that are Aerosol Generating Procedures (AGP) (Cook 2020; Sundaram et al. 2020), necessitating healthcare workers in all disciplines to wear full enhanced Personal Protective Equipment (PPE) during treatment procedures (Heij et al. 2020).

As most dental procedures are elective ones, health authorities around the globe [American Dental Association (ADA), Centers for Disease Control and Prevention (CDC), Royal College of Surgeons of England (RCS), Scottish Dental Clinical Effectiveness Programme (SDCEP) Australian Dental Association (AusDA), and the Ministry of Health and Dental Council New Zealand (NZMOH)] have recommended suspending these procedures (American Dental Association 2020; Centers for Disease Control and Prevention 2020; Royal Collge of Surgeons England 2020; SDCEP 2020; Australian Dental Association 2020; New Zealand Ministry of Health, 2020). The American Academy of Pediatric Dentistry (AAPD) issued re-emergence practice checklist to help prepare paediatric dental practices to start seeing patients (American Academy of Pediatric Dentistry 2020).

Given the aforementioned information and the fact that the literature concerning the impact of COVID-19 on paediatric dentistry is quite limited, this critical review aimed to summarise the international guidelines issued by different authorities regarding the management of the dental environment and patients during the COVID-19 pandemic. The review also aimed to provide a summary of the available non-invasive or minimally invasive caries management strategies that can be utilised by paediatric dentists to minimise AGP in the immediate and near future while dealing with the COVID-19 pandemic.

\section{Materials and methods}

\section{Search strategy}

An evidence-based pertinent literature search of the electronic database; PubMed (MED-LINE), Scopus and Google Scholar was performed to identify the relevant articles and guidelines. The search was conducted up to 6th May 2020 using free text and controlled vocabulary (MeSH) terms. The following keywords were used: "COVID-19," "Corona virus and dentistry," "COVID-19 and Paediatric dentistry guidelines," "Dental management during COVID-19 Pandemic," "Minimal Intervention Dentistry," "Atraumatic Restorative Treatment," and "Post-corona dental management." The electronic search was complemented with a hand search of the following websites: American Dental Association, American Academy of Pediatric Dentistry, European Academy of Paediatric Dentistry, British Society of Paediatric Dentistry, British Society of Periodontology, Australian Dental Association, International Association of Paediatric Dentistry, Scottish Dental Clinical Effectiveness Program, Royal College of Surgeons, Centers for Disease Control and Prevention-USA, and Google Scholar.

\section{Inclusion and exclusion criteria}

The studies were selected if they met the following inclusion criteria: studies in the English language up to 22nd April 2020, randomised or non-randomized clinical trials, prospective or retrospective studies as well as in vivo and in vitro animal studies that reported on the Minimal Intervention Dentistry (MID) as a tool of atraumatic and biological caries management in both primary and permanent dentitions and clinical guidelines on the dental management of paediatric patients during and after the COVID19 pandemic. Exclusion criteria included: studies that used other techniques to treat carious lesions (such as adhesive 
restorations, conventional crowns, pulp therapy) as well as case reports, case series, and review of literature papers. There were no restrictions regarding the date of publication in regards to papers related to MID treatment, but the clinical guidelines on the management of children during COVID-19 were searched between 1st January-6th May 2020 and selected the most updated edition.

\section{Data extraction}

A data extraction form was adopted to collect the following study information: (1) title, (2) authors/guideline, (3) year/month of publication, (4) technique of MID, and (5) subject of the guideline. Papers found in the database and hand searches underwent three rounds of screening before they were included in this update. The first round was a title screen where papers that obviously did not meet the inclusion criteria were excluded. The second was an abstract screen where papers that did not meet the inclusion criteria based on the information provided in the abstract were excluded. These first two stages were carried out by two of the authors. Finally, a full-text screen with a critical appraisal was carried out by all five authors of the manuscript.

\section{Results}

The recommendations from the ADA, CDC, RCS, SDCEP, AusDA, NZMOH and AAPD will be summarised in this section (American Dental Association 2020; Centers for Disease Control and Prevention 2020; Royal Collge of Surgeons England 2020; SDCEP 2020; Australian Dental Association 2020; New Zealand Ministry of Health 2020; American Academy of Pediatric Dentistry 2020).

\section{Triaging dental patients with COVID-19 (phone, tele-health conferences)}

The recommendation from all global healthcare authorities is to triage dental patients either by phone or any other means of tele-health conferencing. The purpose of these triaging procedures is to verify the COVID-19 risk status of the patients and to decide on the urgency of the dental condition. In addition, specific advice for the management of any presenting dental problem and general advice for proper maintenance of oral health can be given.

The AusDA, NZMOH, RCS and SDCEP recommended triaging patients by telephone. Analgesics or antimicrobials, or referral for urgent dental care or emergency care would be recommended as appropriate. Furthermore, the SDCEP and the RCS, divided dental cases into three categories: (1) urgent, (2) emergency and (3) an "advice and self-help" category (SDCEP 2020; Royal College of Surgeons England
2020; Australian Dental Association 2020; New Zealand Ministry of Health 2020). The RCS guidelines included a 'red flag' list comprising children with underlying medical conditions making them more prone to complications if any dental infection is not promptly treated. Consequently, these children should be prioritised for urgent treatment (Royal College of Surgeons England 2020). The CDC recommended telephone triage of all patients in need of emergency dental care. When possible, assessment of patient's dental condition using teleconferencing or tele-dentistry options have been suggested as alternatives to in-office care. If dental treatment can be delayed, offer patients with detailed home care instructions and any appropriate medications (Centers for Disease Control and Prevention 2020). The ADA stated that dentists must "make every effort to interview the patient by telephone, text monitoring system, or video conference before the visit" (American Dental Association 2020).

\section{Definition of urgent and emergency cases}

Table 1 summarises the recommendations in terms of the urgency of the oral health conditions and their management. The ADA guidance was based on the presence/absence of COVID-19 infection symptoms. If an emergency dental patient does not have symptoms consistent with COVID19 infection, they can be seen in dental settings with suitable protocols and PPE in place. However, if there are signs and symptoms of respiratory illness, the patient should be referred for emergency care where appropriate transmissionbased precautions are available.

\section{Medically compromised and special needs children}

Dental management of medically compromised children and children with special needs requires special consideration of their underlying health condition. Dental pain may have a severe impact on these children and their families with evidence of adverse behaviours such as self-harming. Furthermore, children with underlying medical conditions should have special consideration due to the increased risk of developing complications arising from any subsequent infection if the tooth is not treated. These conditions include those with an increased risk of bleeding from medications or health conditions, increased risk of infection (e.g., any immunocompromised state, transplant patient, diabetic, children on immunosuppressants/steroids/chemotherapy), and children at risk of infective endocarditis. Additionally, there are children who are identified as being at a significantly increased risk from COVID-19. These are children with long term respiratory conditions, including chronic lung disease of prematurity with oxygen dependency, cystic fibrosis with significant respiratory problems, childhood interstitial lung disease, severe asthma, and respiratory complications of 


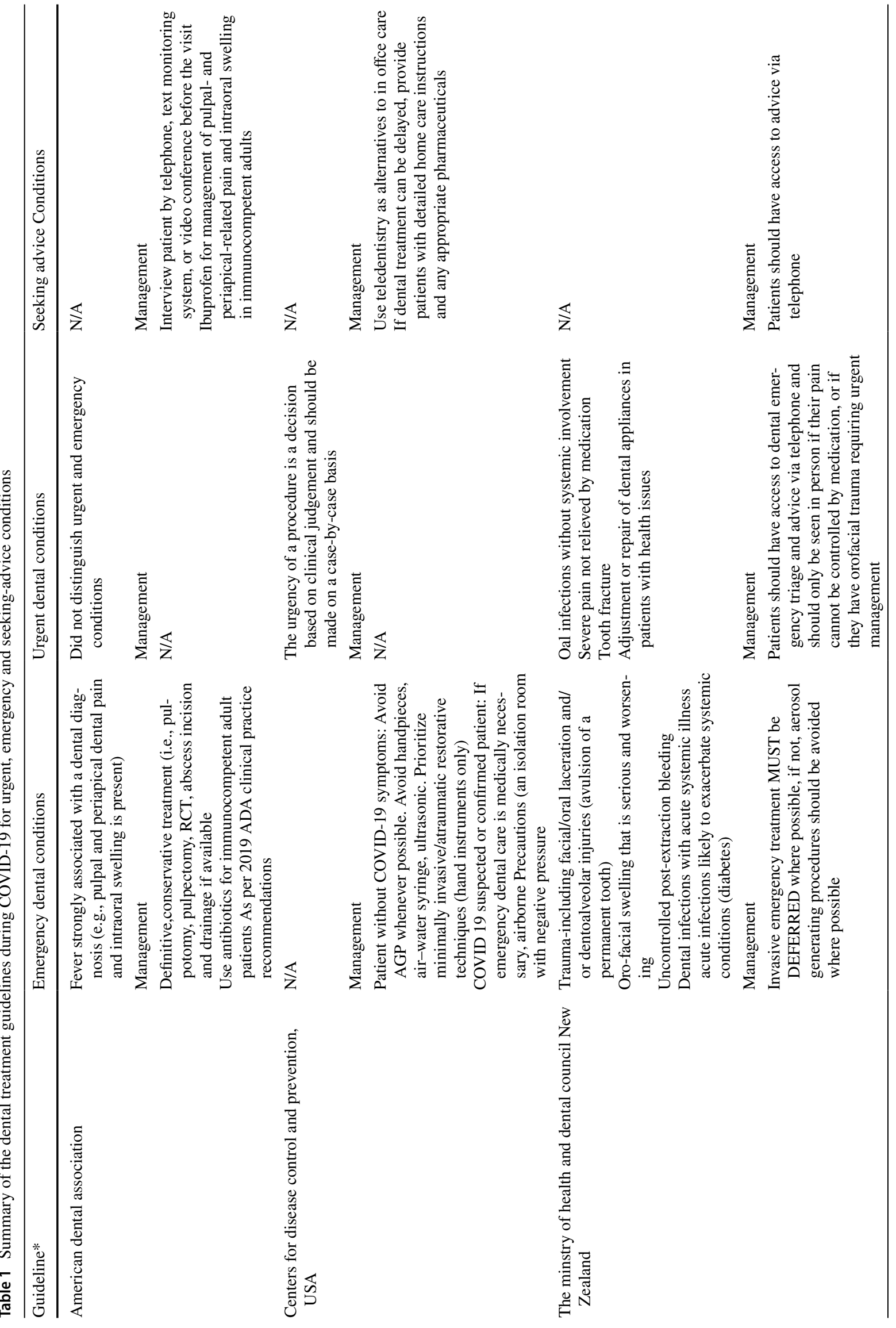




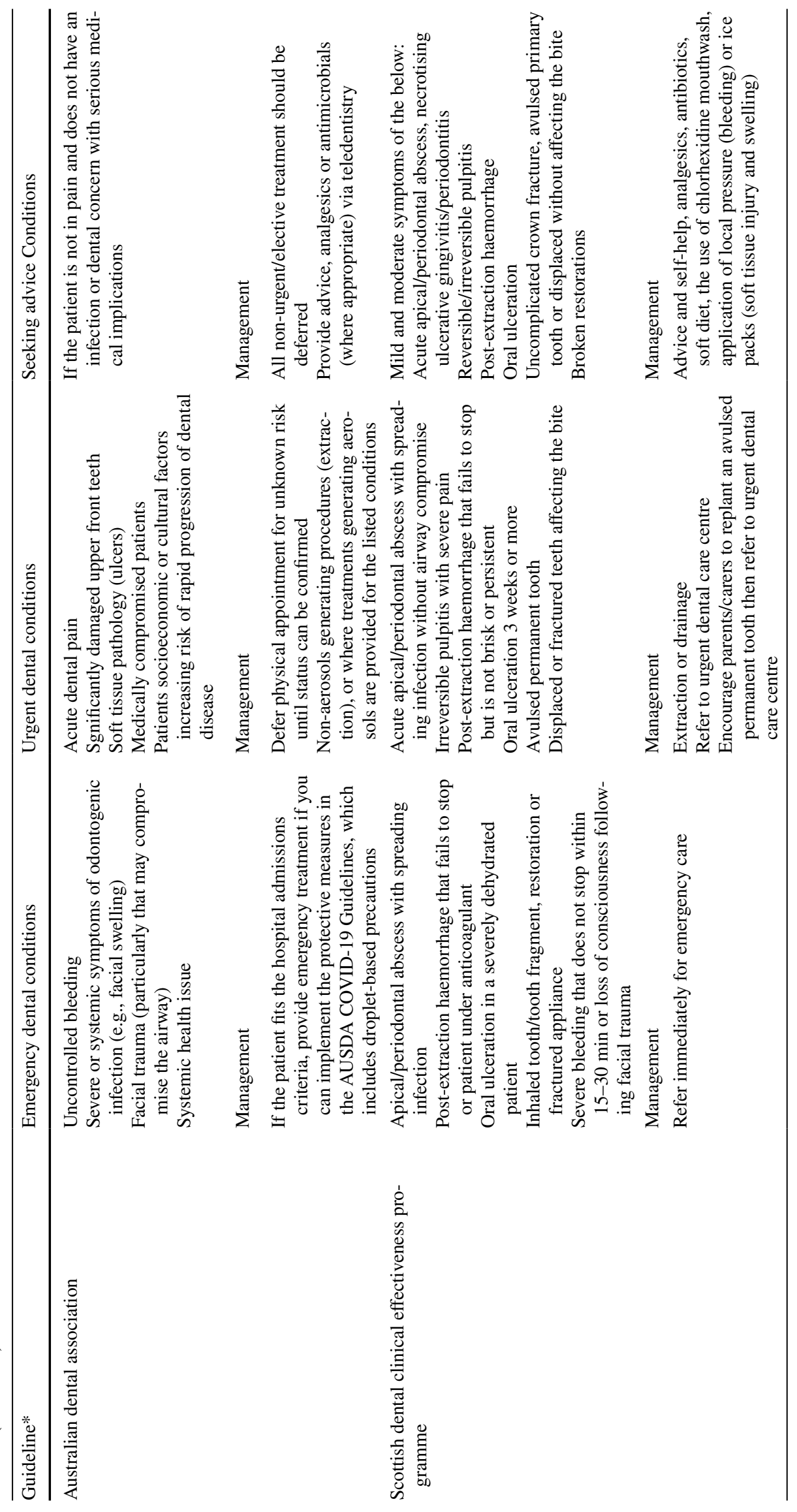




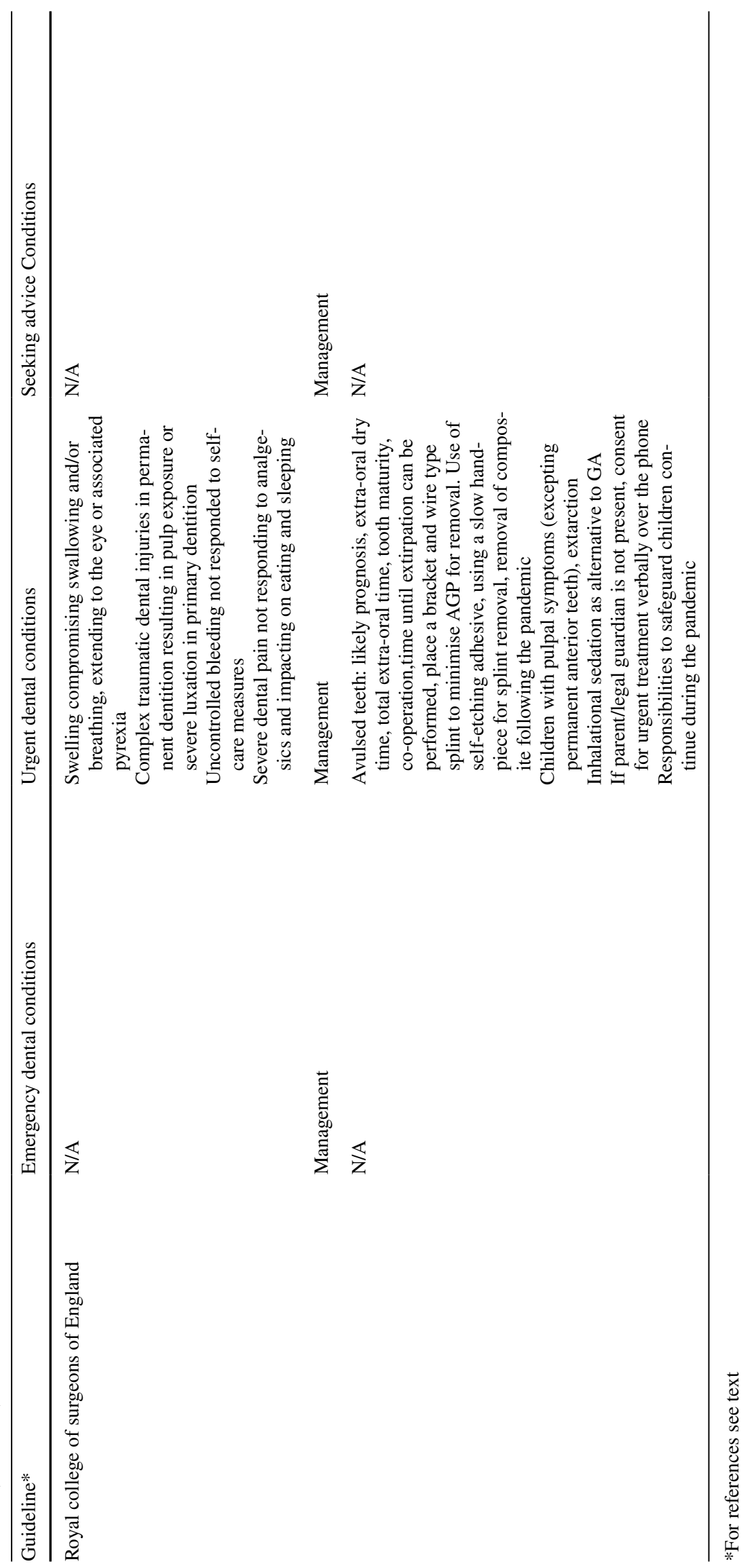


neurodisability. These children should not attend a hospital or dental clinic environment unless the dental condition is considered 'life-threatening' (RCSENG 2018).

The AAPD recommended that to start seeing children with compromised immune illness or with complex medical care needs back in the dental offices, protocols and referral sources should be prepared with hospital protocols readily available. Consultation with the child's physician about the safety of being seen in the dental office or consideration should be given to hospital-based dental clinics if available. Additionally, special consideration should be given to the timing of the appointment in the schedule (1st appointments or special hours/days). If possible, consider building a room that meets hospital standards for infection control and air turnover (American Academy of Pediatric Dentistry 2020).

\section{Access to urgent dental care under general anaesthetic}

Providers have been advised to cancel all elective procedures, including dental treatment under general anaesthesia (GA). It is very crucial to minimise the risk of cross-infection and transmission of the disease. Protocols have been put in place for operating room requirements and preparation for confirmed or suspected COVID-19 patients. The AAPD encouraged to develop a risk-based scale for scheduling general anaesthesia patients (American Academy of Pediatric Dentistry 2020). Where possible, limited emergency provision should be maintained on a regional basis with the following children prioritised for urgent treatment under GA: (RCSEng 2018; Royal College of Surgeons England 2020).

- Sustained trauma to the primary dentition where the child is symptomatic (pain not managed with analgesics, infection not managed with antibiotics or interference with eating), and treatment under local anaesthetic is not possible.

- Trauma to the permanent dentition which needs intervention and treatment under local anaesthetic or sedation is not possible.

- Acute dental infection that is not responsive to antibiotics.

- Intractable pain or discomfort which cannot be managed under local anaesthetic.

- Facial swelling as a result of dental disease and treatment under local anaesthetic is not possible.

- Poor dental health is impacting on or is highly likely to impact on, their medical health and a decision is made that the benefits of surgery outweigh the risks of bringing a child into the hospital during the COVID-19 pandemic.

- Additional needs such as those with learning disability or autism, where dental pain is resulting in self-harm or other disruptive or detrimental behaviours.
- Compromised swallow and are at risk of aspirating a tooth which cannot be removed under local anaesthetic.

\section{Dental environmental and equipment guidelines for COVID-19}

The guidelines issued by the AAPD, ADA, CDC and NZMOH have all included detailed instructions stressing the importance of minimising cross-infection, maintaining the safety of dental healthcare providers, paediatric patients and their guardians, and the proper use of PPE. The ADA guidelines had detailed guidance that should be followed before, during and after the dental care is provided. These guidelines are summarised in Table 2. In addition, Table 2 includes further recommendations for NZMOH regarding the same (American Academy of Pediatric Dentistry 2020; American Dental Association 2020b; Centers for disease control and Prevention 2020; New Zealand Ministry of Health 2020).

A recent Cochrane "rapid' review of international recommendations for the re-opening of dental services from 11 countries reported some common key messages intended to assist policy and decision-makers to produce comprehensive national guidance for their own settings. It was noticed that in the majority of the sources, there was no referenced, underpinning evidence with some areas unlikely to ever have strong (or any) research evidence. Furthermore, there was a highly variable level of detail given across international sources. Filtering facepiece class 2 (FFP2, equivalent to N95) masks are recommended by the majority of international sources for both COVID-19 and non-COVID-19 confirmed cases irrespective of the use of AGPs. A minority of sources recommend the use of a filtering facepiece class 3 (FFP3, equivalent to N99) mask for AGPs (Clarkson et al 2020).

All the guidelines highlighted that during this period, AGP use should be kept to a minimum. Dental caries is best managed by prevention (Toumba et al. 2019). Non-restorative treatment and minimally invasive restorative techniques that generate minimal aerosol are presented in Table 3 and discussed in details in the "Discussion" section.

\section{Discussion}

Dentistry involves many procedures that are, for the most part, AGP and might lead to the spread of the COVID-19 virus to dental healthcare workers and other patients and families. In addition, the wide-spread epidemic has resulted in shortages or critical need for the healthcare PPE (Centers for Disease Control and Prevention 2020). Guidelines issued by different dental and medical organisations attempted to provide clear instructions to dental practitioners regarding prioritising cases seen in the clinics, communicating with 


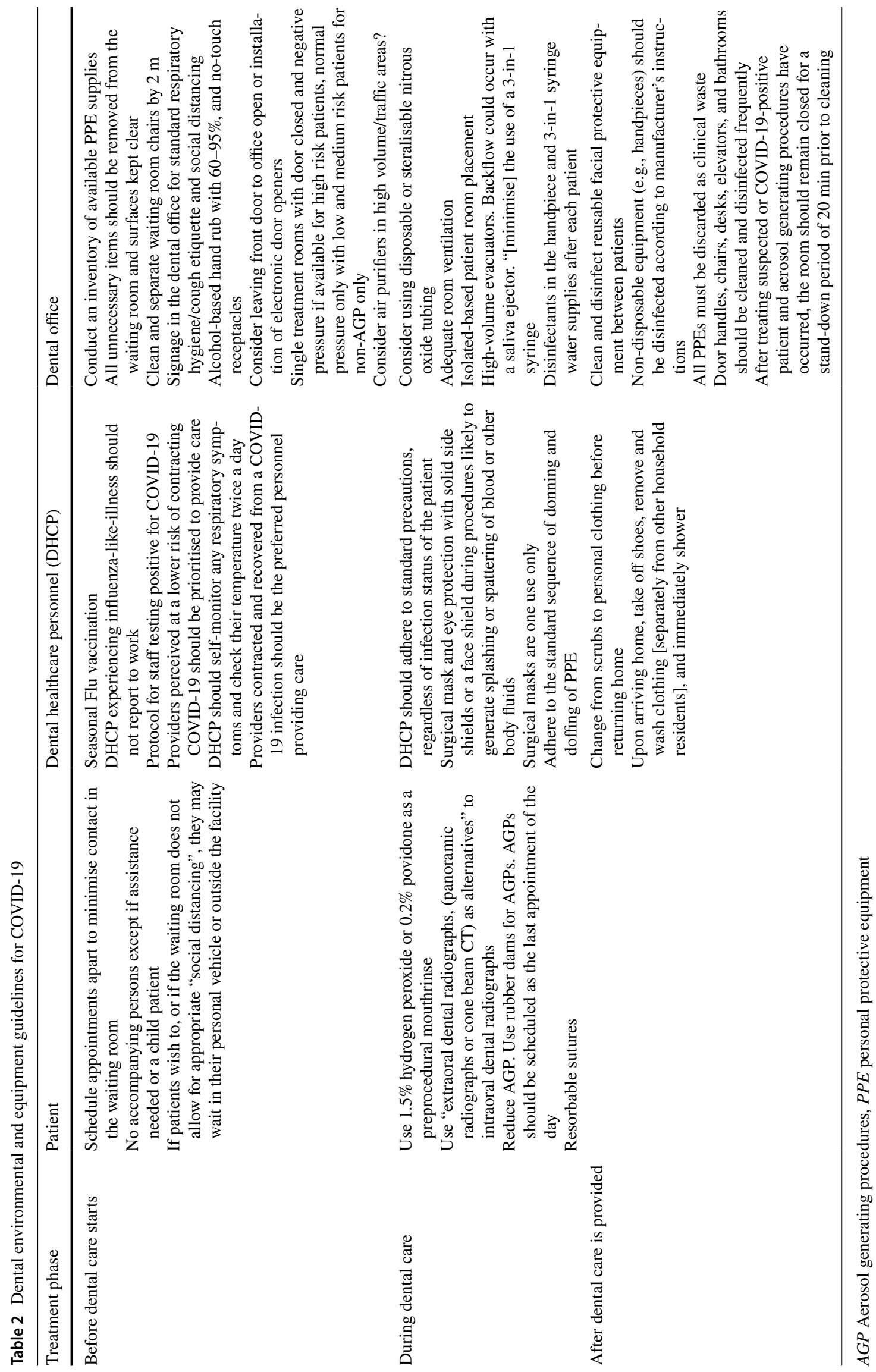


Table 3 Summary of proposed biological caries management techniques and their grade of evidence

\begin{tabular}{|c|c|c|c|c|c|c|}
\hline $\begin{array}{l}\text { Biological Caries } \\
\text { Management Tech- } \\
\text { nique }\end{array}$ & $\begin{array}{l}\text { Cavitated/non- } \\
\text { cavitated lesion }\end{array}$ & $\begin{array}{l}\text { Primary/Permanent } \\
\text { tooth }\end{array}$ & $\begin{array}{l}\text { Symptomatic/ } \\
\text { Asymptomatic } \\
\text { tooth }\end{array}$ & $\begin{array}{l}\text { Proximal/Occlusal } \\
\text { lesion }\end{array}$ & $\begin{array}{l}\text { Grade of evidence } \\
\text { quality }\end{array}$ & $\begin{array}{l}\text { Grade of rec- } \\
\text { ommendation } \\
\text { quality }\end{array}$ \\
\hline Fluoride varnish & Non-cavitated & $\begin{array}{l}\text { Primary and per- } \\
\text { manent }\end{array}$ & Asymptomatic & Proximal & Low to very low & conditional \\
\hline Resin infiltration & Non-cavitated & $\begin{array}{l}\text { Primary and per- } \\
\text { manent }\end{array}$ & Asymptomatic & Proximal & Low to very low & conditional \\
\hline Sealant & Non-cavitated & $\begin{array}{l}\text { Primary and per- } \\
\text { manent }\end{array}$ & Asymptomatic & Occlusal & Moderate & Strong \\
\hline \multirow[t]{2}{*}{ SDF } & Cavitated & Primary & Asymptomatic & $\begin{array}{l}\text { Occlusal and proxi- } \\
\text { mal }\end{array}$ & Moderate & Strong \\
\hline & Cavitated & Permanent & Asymptomatic & $\begin{array}{l}\text { Occlusal and proxi- } \\
\text { mal }\end{array}$ & Low & Conditional \\
\hline \multirow[t]{2}{*}{ Hall PMC } & $\begin{array}{l}\text { Cavitated and non- } \\
\text { cavitated }\end{array}$ & Primary & Asymptomatic & $\begin{array}{l}\text { Occlusal and proxi- } \\
\text { mal }\end{array}$ & High* $^{*}$ & Strong \\
\hline & Cavitated & Permanent & Asymptomatic & $\begin{array}{l}\text { Occlusal and proxi- } \\
\text { mal }\end{array}$ & Low & Conditional \\
\hline \multirow[t]{2}{*}{ ART } & Cavitated & Primary & Asymptomatic & $\begin{array}{l}\text { Occlusal and proxi- } \\
\text { mal }\end{array}$ & Low to very low & Conditional \\
\hline & Cavitated & Permanent & Asymptomatic & $\begin{array}{l}\text { Occlusal and proxi- } \\
\text { mal }\end{array}$ & Low to very low & Conditional \\
\hline ITR & Cavitated & Primary & Asymptomatic & $\begin{array}{l}\text { Occlusal and proxi- } \\
\text { mal }\end{array}$ & Low & Conditional \\
\hline ITR/diagnostic & Cavitated & Primary & $\begin{array}{l}\text { Symptomatic } \\
\text { (reversible pulpi- } \\
\text { tis symptoms) }\end{array}$ & $\begin{array}{l}\text { Occlusal and proxi- } \\
\text { mal }\end{array}$ & Low & Conditional \\
\hline IPC & Cavitated & $\begin{array}{l}\text { Primary and per- } \\
\text { manent }\end{array}$ & $\begin{array}{l}\text { Symptomatic } \\
\text { (reversible pulpi- } \\
\text { tis symptoms) }\end{array}$ & $\begin{array}{l}\text { Occlusal and proxi- } \\
\text { mal }\end{array}$ & Low & Conditional \\
\hline
\end{tabular}

*Cochrane reviews and RCTs

patients through telephone and tele-health conferencing for triaging and recommendation for self-administered actions in cases that can wait to be seen. Guidelines also classified cases into an emergency or urgent ones and "can wait" ones. The guidelines also provided instructions for office preparedness and precautions required for treating patients.

It is appropriate to mention here that the only specific guidelines for the management of paediatric dental cases were issued by the RCS ( Royal College of Surgeons England 2020). AAPD issued guidelines for the preparation of practice to resume clinical activities (American Academy of Pediatric Dentistry 2020). Furthermore, the AAPD issued specific guidance for immediate care for high caries index patients/emergencies encouraging maximising treatment per visit to reduce numbers of visits. However, considerations pertaining to paediatric dentistry and guidance on the management under inhalation sedation or general anaesthetic was only discussed in details by the RCS recommendations for paediatric dentistry during the pandemic. (England Royal College Of Surgeons 2020). The AAPD guidelines only adviced to develop protocols for emergency cases requiring general anaesthesia and to determine when the hospitals/ surgical centres will be scheduling elective dental patients (American Academy of Pediatric Dentistry 2020). Additionally, the SDCEP issued a supplement to their COVID-19 dental guidelines, specifically discussing the pharmacological management of dental emergencies during the pandemic (SDCEP 2020).

The content of guidelines appeared to be quite similar in terms of triaging and classifying oral health care into seeking advice, urgent, and emergency cases especially in the SDCEP, RCS, AusDA and the NZMOH guidelines (SDCEP 2020; Royal College of Surgeons England 2020; Australian Dental Association 2020; New Zealand Ministry of Health 2020).

The guidelines of the AAPD, ADA, CDC and NZMOH included the most details regarding the dental environmental and equipment guidelines for dealing with COVID-19 pandemic. These guidelines went into details regarding the office preparedness to deal with and receive patients during the pandemic compared to the other guidelines quoted in this review (American Dental Association 2020; American Dental Association 2020; Centers for Disease Control and Prevention 2020; New Zealand Ministry of Health 2020). 
Nevertheless, because of impacts on healthcare, poverty and economy, the current practice state of only emergency dental care that many professional organisations have recommended or required in attempts to mitigate the spread of COVID-19 is unsustainable in the long term. For that reason, the AAPD issued a checklist to address significant areas of practice that may need attention prior to the resumption of pre-pandemic levels of care, taking into consideration new and possible changes as a result of the pandemic and its lingering effects (American Academy of Pediatric Dentistry 2020). Additionally, this review attempted to provide "safer" options for paediatric dentists in dealing with their patients during this transitional period.

A 'rapid' Cochrane review for re-opening of dental services recommendations from eleven countries noticed that in the majority of the sources, there was no referenced, underpinning evidence with some areas unlikely to ever have strong (or any) research evidence. Furthermore, there was a highly variable level of detail given across these sources. Most sources recommend avoiding aerosol-generating procedures (AGPs), if possible but still support high-quality clinical care (Clarkson et al. 2020).

\section{Post-COVID-19 era clinical recommendations}

As presented above, the unpredictability of COVID-19 infection in children and the challenges in the diagnosis of symptoms, in addition to the reports of carrier status of the SARS-CoV-2 will present a challenge for all paediatric dentists once normal function in the dental clinics is restored worldwide. Consequently, this may result in increased anxiety for the dental healthcare providers, the parents and the patients. Emphasis should be placed on minimising AGPs as much as possible, and this section will present a summary of the non-restorative and the atraumatic restorative treatment alternatives for caries that are reported in the scientific literature.

\section{Behaviour management}

The first and most important basic technique that all paediatric dentists can utilise to minimise the probability of SARSCoV-2 cross-infection is by appropriate and skillfull behaviour management. Restless, crying children spread more aerosol compared to calm children. We all need to keep in mind the added anxiety that the child might have as a result of the dental healthcare providers having to follow enhanced PPE protocols that may include a face mask. Whenever possible, it is helpful to put this protective equipment on while the child is watching, and while we explain to them in simple terms, the value and use of this equipment. Another suggestion is to have a pin or a sticker that can be disinfected easily with the health provider's photograph displayed over the protective equipment. Having the parents in the dental clinic during treatment might be restricted moving forward. Proper coping techniques and communication between the paediatric dentist and the parents and their child are very essential. The AAPD recommends delaying seeing pediatric patients who require physical behaviour management, considering treating patients while on parent's lap with parent wearing a mask and having passed negative screening criteria, dedicating special hours in the day for such patients (American Academy of Pediatric Dentistry 2020).

\section{Prevention}

Dental caries is largely preventable, and effective evidencebased strategies are available for caries prevention as well as management of the disease. Patient-centred enhanced prevention plans tend to be more effective. (SDCEP 2018). Therefore, it is only logical to prioritise and emphasise all oral health preventive and therapeutic measure during this time (Toumba et al. 2019).

\section{Biological caries management techniques}

Using biological atraumatic, non-invasive or minimally invasive treatment methods that require minimal or no AGP would be more logical and safer given the high success rate of the biological approach in caries management (BaniHani et al. 2018). The use of evidence-based biological approaches for caries management in both primary and permanent dentition might be more appropriate under the current and near-future circumstances of COVID-19.

A 2016 European Academy of Paediatric Dentistry policy document regarding best clinical practice guidance for management of early caries lesions in children and young adults concluded that non-cavitated caries lesions can be managed non-invasively in the majority of cases. The spectrum of measures includes a low cariogenicity tooth-friendly diet, daily and appropriate management of the biofilm, home and within the dental office/surgery usage of fluorides as well as sealing techniques. The policy emphasised on the importance of performing caries risk assessment at a child's first dental visit, and reassessments on a regular basis (Kühnisch et al. 2016).

Biological treatment alternatives for asymptomatic teeth or teeth exhibiting signs of reversible pulpitis include sealing non-cavitated caries, using fluoride varnish and resin infiltration to arrest non-caviateted caries, atraumatic or alternative restorative technique (ART), interim therapeutic restorations (ITR) indirect pulp capping (IPC), the Hall technique (HT); and the use of Silver Diamine Fluoride (SDF) (Slayton et al. 2018; Urquhart et al. 2019; Al Halabi et al. 2018; Tedesco et al. 2017; American Academy of Pediatirc Dentistry 2017a, b; Hussein et al. 2020; Seifo et al. 2018). 
These aformentioned biological techniques are summarised in Table 3, along with the strength of supportive evidence.

\section{Sealants}

To arrest or reverse the non-cavitated carious lesions on the occlusal surfaces of both primary and permanent teeth, the use of a combination of sealants and $5 \% \mathrm{NaF}$ varnish (application every 3-6 months) or sealants alone over $5 \% \mathrm{NaF}$ varnish alone (application every 3-6 months) is recommended by the ADA (Slayton et al. 2018; Urquhart et al. 2019).

In addition, sealing dental caries in permanent teeth where the carious lesion is partially removed or completely left has been employed as an accepted therapeutic technique by some researchers with success rates up to ten years. Furthermore, in permanent teeth, the number of bacteria detected after conventional complete caries removal was higher than that which remained in sealed carious lesions (Maltz et al. 2012). Thus, this practice may be adopted in the post-COVID-19 era.

For approximal surfaces, ADA suggests, to arrest or reverse non-cavitated carious lesions of primary and permanent teeth, clinicians should use 5\% NaF varnish (application every 3-6 months), resin infiltration alone, resin infiltration plus 5\% NaF varnish (application every 3-6 months), or sealants alone (Slayton et al. 2018; Urquhart et al. 2019).

\section{Atrumatic restorative technique (ART)}

ART has been endorsed by the World Health Organization (WHO) as a means of restoring and preventing caries in populations with limited access to traditional dental care (Tedesco et al. 2017). The use of ART for both primary and permanent teeth in children presents a valid option to manage caries successfully. In addition, glass ionomer ART sealants can also be used as a treatment option. A recent systematic review and meta-analysis on survival percentages of ART restorations and sealants in posterior teeth concluded that ART single-surface restorations presented high survival percentages in both dentitions compared to multiple-surface restorations. In addition, ART sealants presented a high-caries-preventive effect (de Amorim et al. 2018). Thus, ART presents as an invaluable, effective evidence-based alternative to conventional methods in the post-COVID-19 era for treating and preventing carious lesions in posterior teeth. Nevertheless, a Cochrane Database of Systematic Review concluded that low-quality evidence suggested that ART use may have a higher risk of restoration failure than conventional treatment for caries lesions in primary teeth. The effects of ART using composite and resin-modified glass ionomer cement (RM-GIC) are uncertain due to the very low quality of the evidence, and we cannot rely on the findings (Dorri et al. 2017).

\section{Interim therapeutic restorations (ITR)}

Another non-AGP method is the interim therapeutic restoration as defined by the AAPD which utilises similar techniques to ART but has different therapeutic goals. ITR more accurately describes the procedure used in contemporary dental practice in the USA. It may be used to restore and prevent further decalcification and caries in young patients, uncooperative patients, or patients with special health care needs. The technique can also be used when conventional cavity preparation and/or placement of conventional dental restorations are not feasible and dental care needs to be postponed (American Academy of Pediatric Dentistry 2017a).

A suggested modified application of ITR is the step-wise excavation in children with multiple open carious lesions prior to definitive restoration of the teeth. (Coll et al. 2013) The ITR procedure involves removal of caries using hand or slow speed rotary instruments with caution not to expose the pulp. Leakage of the restoration can be minimised with maximum caries removal from the periphery of the lesion. Following preparation, the tooth is restored with an adhesive restorative material such as self-setting or RM-GIC (Berg, 2002).

Coll et al. (2013) investigated the use of ITR in deep carious lesions as a diagnostic tool for pulpal status. They indicated that glass ionomer cement (GIC) used as an ITR for a period of one to $\geq$ three months as a diagnostic tool on asymptomatic primary molars has significantly improved the success rate of vital pulp therapy (Coll et al. 2013). The ITR placed in proximal lesions significantly improved the success rate of vital pulp therapy done subsequently, while ITR placed in non-proximal lesions did not have any significant effect on the success rate. No difference was found in the success rate of indirect pulp capping and vital formocresol pulpotomy treatment after the ITR. Therefore, one can see that the use of ITR in the current COVID-19 pandemic and beyond is a potential valid dental tool.

\section{The Hall technique}

The HT is a non-surgical/non-invasive method used to restore a carious but asymptomatic and non-septic primary molar in a child using a child-friendly approach. As it is a non-AGP procedure, it may be valid and relevant to report it here (Hussein et al. 2020). It relies on sealing non-pulpally involved carious lesions on primary molars in situ using a preformed metal crown (PMC) and GIC (Welbury 2017). The HT manual recommends crowning primary molars that have no signs of irreversible pulpitis with clear radiographic evidence of a band of normal dentine between the pulp space and the carious lesions. The PMC is placed on the affected molar after space is created using orthodontic spacers/separators, which are applied for 3-5 days. No local anaesthesia 
is utilised, and no attempt to surgically remove the carious tissue is made (Innes et al. 2015a).

The evidence-based platform that rolled out the HT was clearly sound and was supported by a randomised controlled trial and a Cochrane review (Santamaria et al. 2018; Innes et al. 2015b). Controversial issues like the effect of the PMC placed using the HT on the occlusion because of the slight initial opening of the bite was found to resolve spontaneously after a month (van der Zee V 2010). A recent pilot study investigated the effect of the Hall technique crown application on the masseter muscle activity. The study concluded that Hall technique crowns might affect masseter muscle activity in children immediately post-treatment, but the activity returned to and later exceeded baseline levels at 2-6 weeks, respectively, while the rest masseter muscle activity remained unchanged (Abu Serdaneh et al. 2020). In addition, the HT was found to be more cost-effective than the conventional surgical method of treatment. It is now considered in some circles "the golden standard" for managing the multi-surface non-pulpally involved carious primary molars (Deery 2015). According to a review by Welbury (2017), the HT is an ideal restoration for a primary tooth in a young child as it is quick and easy for the child to cope with. It does not require local anaesthesia and has proven efficacy by means of randomised clinical trials (Welbury 2017).

A published case series reported the success of combining the use of GIC ITR as a diagnostic tool for teeth with deep caries that are asymptomatic or exhibiting symptoms of reversible pulpitis with normal radiographic appearance (Al Halabi et al. 2018; Coll et al. 2013). This was followed by the use of the HT technique PMC after a period of time. The confirmation of a diagnosis of reversible pulpitis is demonstrated by the absence of signs and symptoms.

Taking into account the methods highlighted above, a multicentre retrospective evaluation of outcomes of both the biological method of treatment represented by the HT and IPT and the conventional method of complete caries removal and or pulpotomies concluded that both the conventional and biological treatment approaches had similar outcomes and were equally successful for the management of carious lesions in the primary dentition (BaniHani et al. 2018). Thus, this helps in supporting the debate that negates the use of AGP wherever possible under the COVID-19 situation.

What about permanent teeth? The HT is used mainly as a biological restoration for primary molars. However, it has been suggested by the caries management guidelines of the SDCEP as a treatment option to keep badly broken down first permanent molars (FPMs) free from symptoms until the ideal age for extractions (SDCEP 2018), although there is no robust supportive evidence. Millar et al. (2015) stated that PMCs are beneficial for intermediate-term management of hypomineralised or hypoplastic FPMs with enamel breakdown, caries and sensitivity (Millar et al. 2015). They concluded that HT could be used even for anxious children because of its simplicity of avoiding injections and drilling. Although the data supporting the use of HT as a biological treatment option for FPMs is scarce, we suggest its use as an option in post-COVID-19 era to avoid drilling required for conventional restorations, thus reducing AGP.

\section{Silver diamine fluoride (SDF)}

Non-restorative caries control has had its major supporters for a while now, but the new emphasis in the current and post-COVID-19 period is essential. The use of SDF should be highlighted here (Kidd 2012; Seifo et al. 2020). SDF is a clear, odourless liquid indicated for desensitisation of noncarious tooth lesions and molar incisor hypomineralisation of FPMs. It can also be used for arresting carious lesions in high caries-risk children, children who are difficult to control, progressing carious lesions, unable to tolerate invasive treatment, and those who are medically compromised or have additional care needs. (Innes et al. 2019). The ADA recommends using SDF to arrest advanced cavitated carious lesions on any coronal surface of primary and permanent teeth. Their expert panel suggests clinicians prioritise the use of 38\% SDF solution (biannual application) over 5\% NaF varnish (Slayton et al. 2018; Urquhart et al. 2019).

According to the recent umbrella review by Seifo et al. (2019), systematic reviews consistently supported SDF's effectiveness for arresting coronal caries in the primary dentition. There is insufficient evidence to draw conclusions on SDF use in children for the prevention of caries in primary and permanent dentition. No serious adverse events were reported. (Seifo et al. 2019).

\section{Conclusion}

- COVID-19 has and will continue to have a major impact on the practice of paediatric dentistry. Traiging cases into advice only, urgent care and emergency cases should become standard practice in the current pandemic. Avoidance of elective AGPs is recommended wherever possible and management of emergencies should take priority.

- Once the current worldwide restrictions are eased, we might modify some approaches in our practice of paediatric dentistry utilising more prevention-centred practices and/or atraumatic non or minimally invasive techniques in caries management. 


\section{Compliance with ethical standards}

Conflict of interest The authors declare that they have no conflict of interest.

\section{References}

Abu Serdaneh S, Alhalabi M, Kowash M, et al. Hall technique crowns and children's masseter muscle activity: a surface electromyography pilot study. Int J Paediatr Dent. 2020;30(3):303-13.

Al Halabi M, Kowash M, Hussein I. Hybrid child-friendly biological primary molar restorative alternatives to general anaesthesia. Dent Update. 2018;2018(45):728-41.

American academy of pediatric dentistry 2020. A guide for re-entry into practice for pediatric dentists during the COVID-19 pandemic. https://www.aapd.org/about/about-aapd/news-room/covid -19/. Accessed 28 Apr 2020.

American academy of pediatric dentistry. Use of vital pulp therapies in primary teeth with deep caries lesions. Pediatr Dent. 2017a;2017(39):146-159.

American academy of pediatric dentistry council on clinical affairs. Policy on interim therapeutic restorations (ITR). Pediatr Dent. 2017b;39(6):64-5 .

American dental association 2020. ADA interim guidance for minimizing risk of COVID-19 transmission. Available: https://www. ada.org/en/publications/ada-news/2020-archive/april/ada-relea ses-interim-guidance-on-minimizing-covid-19-transmission-riskwhen-treating-emergencies. Accessed 15 Apr 2020.

Australian dental association 2020. ADA dental service restriction in COVID-19. Available: https://adawa.com.au/covid-19-updates/. Accessed 15 Apr 2020

Banihani A, Duggal M, Toumba J, Deery C. Outcomes of the conventional and biological treatment approaches for the management of caries in the primary dentition. Int J Paediatr Dent. 2018;28:12-22.

Berg JH. Glass ionomer cements. Pediatr Dent. 2002;24:430-8.

Cao Q, Chen YC, Chen CL, Chiu CH. SARS-CoV-2 infection in children: transmission dynamics and clinical characteristics. J Formos Med Assoc. 2020;119:670-3.

Centers for disease control and prevention 2020. Interim guidance for businesses and employers to plan and respond to coronavirus disease 2019 (COVID-19). Available: https://www.cdc.gov/coron avirus/2019-ncov/community/guidance-business-response.html. Accessed 15 Apr 2020.

Choi SH, Kim HW, Kang JM, Kim DH, Cho EY. Epidemiology and clinical features of coronavirus disease 2019 in children. Clin Exp Pediatr. 2020;63(4):125-32.

Clarkson J Ramsay C Aceves M et al. Recommendations for the reopening of dental services: a rapid review of international sources. Cochrane Oral Health, 6 May 2020. https://oralhealth.cochrane. $\mathrm{org} /$ news/recommendations-re-opening-dental-services-rapid -review-international-sources. Accessed 9 May 2020.

Coll JA, Campbell A, Chalmers NI. Effects of glass ionomer temporary restorations on pulpal diagnosis and treatment outcomes in primary molars. Pediatr Dent. 2013;35:416-21.

Cook TM. Personal protective equipment during the coronavirus disease (COVID) 2019 pandemic a narrative review. Anaesthesia. 2020;75(7):920-7.

Cruz AT, Zeichner SL. COVID-19 in children: initial characterization of the pediatric disease. Pediatrics. 2020;145(6):e20200834. https ://doi.org/10.1542/peds.2020-0834.

De Amorim RG, Frencken JE, Raggio DP, Chen X, Hu X, Leal SC. Survival percentages of atraumatic restorative treatment
(ART) restorations and sealants in posterior teeth: an updated systematic review and meta-analysis. Clin Oral Investig. 2018;22:2703-25.

Deery C. The hall technique: a paradigm shift in our care of children with caries. Dent Update. 2015;42:903-4.

Dong Y, Mo X, Hu Y, et al. Epidemiology of COVID-19 among children in China. Pediatrics. 2020;145(6):e20200702. https://doi. org/10.1542/peds.2020-0702.

Dorri M, Martinez-Zapata MJ, Walsh T, Marinho VCC, Sheiham A, Zaror C. Atraumatic restorative treatment versus conventional restorative treatment for managing dental caries. Cochrane Database Syst Rev 2017;2017(12):CD008072. https://doi. org/10.1002/14651858.CD008072.pub2.

Ebrahim SH, Memish ZA. COVID-19 - the role of mass gatherings. Travel Med Infect Dis. 2020;34:101617. https://doi.org/10.1016/j. tmaid.2020.101617.

Frauenfelder C, Butler C, Hartley B, Cochrane L, Jephson C, Nash R, Hewitt R, Albert D, Wyatt M, Hall A. Practical insights for paediatric otolaryngology surgical cases and performing microlaryngobronchoscopy during the COVID-19 pandemic. Int $\mathrm{J}$ Pediatr Otorhinolaryngol. 2020;2020(134):110030. https://doi. org/10.1016/j.ijporl.2020.110030.

Heij R, Steel AG, Young PJ. Testing for coverage from personal protective equipment. Anaesthesia. 2020;75(7):966-7.

Hussein I, Al Halabi M, Kowash M, et al. Use of the Hall technique by specialist paediatric dentists: a global perspective. Br Dent J. 2020;228(1):33-8.

Innes N, Evans D. The Hall technique: a minimal intervention, child centred approach to managing the carious primary molar. a user manual. University of Dundee. 2015a. https://dentistry.dundee.ac. uk/files/3M_93C\%2520HallTechGuide2191110.pdf. Accessed 27 Apr 2020.

Innes NP, Ricketts D, Chong LY, Keightley AJ, Lamont T, Santamaria RM. Preformed crowns for decayed primary molar teeth. Cochrane Database Syst Rev 2015b;31(12):CD005512. https:// doi.org/10.1002/14651858.CD005512.pub3.

Innes NPT, Chu CH, Fontana M, Lo ECM, Thomson WM, Uribe S, Heiland M, Jepsen S, Schwendicke F. A century of change towards prevention and minimal intervention in cariology. J Dent Res. 2019;98:611-7.

Jiang X, Rayner S, Luo MH. Does SARS-CoV-2 has a longer incubation period than SARS and MERS? J Med Virol. 2020;92:476-8.

Kidd E. Should deciduous teeth be restored? Reflections of a cariologist. Dent Update. 2012;39(3):159-62,165-6. https://doi. org/10.12968/denu.2012.39.3.159.

Kühnisch J, Ekstrand KR, Pretty I, et al. Best clinical practice guidance for management of early caries lesions in children and young adults: an EAPD policy document. Eur Arch Paediatr Dent. 2016;17(1):3-12.

Maltz M, Henz SL, De Oliveira EF, Jardim JJ. Conventional caries removal and sealed caries in permanent teeth: a microbiological evaluation. J Dent. 2012;40:776-82.

Mccloskey B, Zumla A, Ippolito G, Blumberg L, Arbon P, Cicero A, Endericks T, Lim P, Borodina L, M., and Group, W. H. O. N. C.-M. G. E. Mass gathering events and reducing further global spread of COVID-19: a political and public health dilemma. Lancet. 2020;395:1096-9.

Millar LM, Cairns AM, Fowler L. Preformed metal crowns for the permanent dentition. Prim Dent J. 2015;4:44-5.

New Zealand Ministry Of Health 2020. Guidelines for oral health services at COVID-19 Alert Level 4. New Zealand dental association. Available: https://www.nzda.org.nz/covid-19/protecting -your-health. Accessed 15 Apr 2020.

Pavone P, Ceccarelli M, Taibi R, La Rocca G, Nunnari G. Outbreak of COVID-19 infection in children: fear and serenity. Eur Rev Med Pharmacol Sci. 2020;24(8):4572-5. 
Qiu H, Wu J, Hong L, Luo Y, Song Q, Chen D. Clinical and epidemiological features of 36 children with coronavirus disease 2019 (COVID-19) in Zhejiang, China: an observational cohort study. Lancet Infect Dis. 2020;S1473-3099(20):30198-205.

Rabaan AA, Al-Ahmed SH, Haque S, Sah R, Tiwari R, Malik YS, Dhama K, Yatoo MI, Bonilla-Aldana DK, Rodriguez-Morales AJ. SARS-CoV-2, SARS-CoV, and MERS-COV: a comparative overview. Infez Med. 2020;28:174-84.

RCSENG. 2018. Number of children aged 5 to 9 admitted to hospital due to tooth decay rises again. The Royal College of Surgeons of England. Available: https://www.rcseng.ac.uk/news-and-events/ media-centre/press-releases/hospital-admission-tooth-decay/. Accessed 20 Apr 2020.

Rothan HA, Byrareddy SN. The epidemiology and pathogenesis of coronavirus disease (COVID-19) outbreak. J Autoimmun. 2020;109:102433.

Royal college of surgeons England 2020. Recommendations for paediatric dentistry during COVID-19 pandemic. https://www.rcsen g.ac.uk/-/media/files/rcs/fds/guidelines/paediatric-dentistry-covid 19.pdf. [Acessed 20/4/ 2020]

Rubino S, Kelvin N, Bermejo-Martin JF, Kelvin D. As COVID-19 cases, deaths and fatality rates surge in Italy, underlying causes require investigation. J Infect Dev Ctries. 2020;14:265-7.

Santamaria RM, Innes NPT, Machiulskiene V, Schmoeckel J, Alkilzy $\mathrm{M}$, Splieth CH. Alternative caries management options for primary molars: 2.5-year outcomes of a randomised clinical trial. Caries Res. 2018;51:605-14.

SDCEP 2018. Prevention and manamgement of dental caries in children. https://www.sdcep.org.uk/wp-content/uploads/2018/05/ SDCEP-Prevention-and-Management-of-Dental-Caries-in-Child ren-2nd-Edition.pdf. Accessed 20 Apr 2020.

SDCEP. SDCEP COVID-19 guidance update. Br Dent J. 2020;228:496.

Seifo N, Al-Yaseen W, Innes N. The efficacy of silver diamine fluoride in arresting caries in children. Evid Based Dent. 2018;19:42-3.

Seifo N, Cassie H, Radford JR, Innes NPT. Silver diamine fluoride for managing carious lesions: an umbrella review. BMC Oral Health. 2019;19:145.

Seifo N, Robertson M, Maclean J, Blain K, Grosse S, Milne R, Seeballuck $\mathrm{C}$, Innes $\mathrm{N}$. The use of silver diamine fluoride (SDF) in dental practice. Br Dent J. 2020;228:75-81.

Shen Q, Guo W, Guo T, et al. Novel coronavirus infection in children outside of Wuhan China. Pediatr Pulmonol. 2020;55(6):1424-9.

Sinha IP, Harwood R, Semple MG, et al. COVID-19 infection in children. Lancet Respir Med. 2020;8(5):446-7. https://doi. org/10.1016/S2213-2600(20)30152-1.

Slayton R1, Urquhart O, Araujo M, et al. Evidence-based clinical practice guideline on nonrestorative treatments for carious lesions: a report from the American dental association. J Am Dent Assoc. 2018;149(10):837-849.e19.

Snyder MR, Ravi SJ. 1818, 1918, 2018: Two centuries of pandemics. Health Secur. 2018;16(6):410-5.
Sundaram M, Ravikumar N, Bansal A, et al. Novel coronavirus 2019 (2019-ncov) infection: part II: respiratory support in the pediatric intensive care unit in resource-limited settings. Indian Pediatr. 2020;57(4):335-42.

Su L, Ma X, Yu H, Zhang Z, Bian P, Han Y, Sun J, Liu Y, Yang C, Geng J, Zhang Z, Gai Z. The different clinical characteristics of corona virus disease cases between children and their families in Chin: the character of children with COVID-19. Emerg Microbes Infect. 2020;9:707-13.

Tedesco TK, Calvo AF, Lenzi TL, Hesse D, Guglielmi CA, Camargo LB, Gimenez T, Braga MM, Raggio DP. ART is an alternative for restoring occlusoproximal cavities in primary teeth - evidence from an updated systematic review and meta-analysis. Int J Paediatr Dent. 2017;27:201-9.

Toumba KJ, Twetman S, Splieth C, Parnell C, van Loveren C, Lygidakis NA. Guidelines on the use of fluoride for caries prevention in children: an updated EAPD policy document. Eur Arch Paediatr Dent. 2019;20(6):507-516.

Urquhart O, Mp T, Pilcher L, et al. Nonrestorative treatments for caries: systematic review and network meta-analysis. J Dent Res. 2019;98(1):14-26.

Van Der Zee V, Van Amerongen VAW. Influence of preformed metal crowns (hall technique) on the occlusal vertical dimension in the primary dentition. Eur Arch Paediatr Dent. 2010;11:225-7.

Welbury RR. The hall technique 10 years on: its effect and influence. Br Dent J. 2017;222:421-2.

Wu C, Chen X, Cai Y, et al. Risk factors associated with acute respiratory distresssyndrome death in patients with coronavirus disease 2019 pneumonia in Wuhan, China. JAMA Intern Med. 2020. https ://doi.org/10.1001/jamainternmed.2020.0994.

Xia W, Shao J, Guo Y, Peng X, Li Z, Hu D. Clinical and CT features in pediatric patients with COVID-19 infection: different points from adults. Pediatr Pulmonol. 2020;55(5):1169-74.

Yang Y, Peng F, Wang R, Guan K, Jiang T, Xu G, Sun J, Chang C. The deadly coronaviruses: the 2003 SARS pandemic and the 2020 novel coronavirus epidemic in China. J Autoimmun. 2020;109:102434.

Yu F, Du L, Ojcius DM, Pan C, Jiang S. Measures for diagnosing and treating infections by a novel coronavirus responsible for a pneumonia outbreak originating in Wuhan China. Microbes Infect. 2020;22:74-9.

Zimmermann P, Curtis N. Coronavirus infections in children including COVID-19 an overview of the epidemiology, clinical features, diagnosis, treatment and prevention options in children. Pediatr Infect Dis J. 2020;39(5):355-68.

Publisher's Note Springer Nature remains neutral with regard to jurisdictional claims in published maps and institutional affiliations. 